\title{
On the microscopic theory of the exciton ring fragmentation
}

\author{
A.V. Paraskevov and T.V. Khabarova \\ Russian Research Center "Kurchatov Institute", Kurchatov Sq. 1, Moscow 123182, Russia
}

\begin{abstract}
The description is presented for the dependence of the indirect exciton condensate density at the ring as a function of the polar angle at zero temperature with the involvement of the processes of formation and recombination of the excitons. In particular, starting from the quasi one-dimensional Gross-Pitaevskii equation with a spatially uniform generating term, we derive an exact analytical solution yielding the fragmentation of an exciton ring which is probably observed in the experiments.
\end{abstract}

PACS numbers: 71.35.-y, 71.35.Lk, 03.75.Hh

Keywords: Indirect excitons; Bose-Einstein condensation; Pattern formation

1. Introduction. In the recent experiments of the Butov's group [1, 2] the macroscopically ordered coherent state (MOCS) of the indirect exciton system has been observed in the coupled quantum wells. The state represents a periodic fragmentation of the ring formed in the intersection domain of the surplus densities of electrons in one well and photoexcited holes in another [3, 4, , 5]. The fragmentation is observed below some critical temperature $\left(T_{c} \sim 1 K\right)$ and within a certain range of the laser pumping power when the density $n$ of indirect excitons (IEs) at the ring is sufficiently large and the excitons are well defined $\left(n \sim 10^{9} \mathrm{~cm}^{-2}\right.$, an average spacing between IEs at the ring is $\bar{a}_{e x-e x} \sim n^{-1 / 2} \sim 0.1 \mu m$, the IE Bohr radius is $a_{B} \sim 10^{-2} \mu m, \bar{a}_{e x-e x} / a_{B} \sim 10$ ). The typical parameters of the fragmented ring are as follows [2]: average ring radius is $R \sim 10^{2} \mu \mathrm{m}$, the ring width is $\Delta R \sim 10 \mu \mathrm{m}$, the average radius of a fragment is $r_{f r} \approx \Delta R / 2$, and a number of fragments on the ring is $N_{f r} \approx 50$. The evidence for the MOCS coherence results from a clear interference pattern of the IE recombination radiation from one of the ring fragments [6].

At present, there exist two different phenomenological theories of the MOCS formation. The first theory [7] is based on the assumption that the IE system represents a degenerate two-dimensional quasi-ideal Bose gas and the rate of aggregating an electron and a hole to form an indirect exciton is proportional to the factor $\left(1+N_{0}\right)$, where $N_{0}$ is the number of the IEs in the zero momentum state. Under definite conditions this leads to the instability against fluctuations of the IE ring density. However, the theory seems to be intrinsically inconsistent since, on the one hand, the dipole-dipole interaction between the excitons is neglected, what is allowable only in the low density limit, and, on the other hand, the exciton condensate density should be sufficiently large so that the contribution from the stimulated processes would be essential.

The second theory [8] is based on that the total contribution of the repulsive dipole-dipole and attractive van der Waals interactions becomes attractive at the distances smaller than several IE radii. According to this

\footnotetext{
*E-mail address: paraskev@kiae.ru
}

theory, the ring fragmentation is due to formation of the islands of electron-hole liquid and is not associated with the Bose-Einstein condensation of the IEs. (We also mention paper [9] in which the IE energy distribution is suggested to be the Boltzmann one and, along with an attractive pair potential, the repulsive three-body interaction between IEs is considered. At the same time the nature of the latter is not explained.) However, the results of the experiment [10] unambiguously evidence for the repulsive character of the IE interaction. This is consistent with the idea that just this interaction results in a rapid IE thermalization (along with the nonconservation of a transverse momentum) and in the screening of the surface defects in the quantum wells plane. Note that any extension beyond the dipole approximation while considering the interaction between the IEs is equivalent to the deviation from the Bose statistics, which should selfconsistently be taken into account.

As is seen from the pictures of the spatial distribution of the IE luminescence (see [1, 2]), there exist two different spatial periods on the fragmented ring, namely, the size of a fragment and the size of a dark region separating adjacent fragments. Therefore, it is clear that the density as a function of the polar angle should be expressed via a periodic function with nonsymmetric half-periods, i.e., an elliptic function. So, if $\rho(\phi)$ is an elliptic function, it satisfies equation $\left(\rho_{\phi}^{\prime}\right)^{2}=P_{4}(\rho)$, where $P_{4}(\rho)$ is a quartic polynomial in $\rho$. To the same form one can reduce the one-dimensional stationary Gross-Pitaevskii equation without an external potential, which, as is well known, describes a condensate of Bose particles. Hence, the idea arises that the ring fragmentation can be explained assuming that under experimental conditions an IE condensate exists on the ring. In addition, the formation and recombination of excitons on the ring can be taken into account by their interaction with some scalar generating, or "source-drain", field independent of coordinates. Formally, this is similar to the contribution from the dipole interaction of atoms with the electromagnetic field. If the number of the IEs on the ring does not vary in time, a concept of energy of the system is meaningful and one can find the stationary states of the condensate as well as the corresponding density spatial distributions.

In this Letter, we suggest the simplest microscopic the- 
ory of the MOCS based on the thing that the presence of BEC in the IE system (in spite of the BEC depletion due to repulsive exciton-exciton interaction) as well as the influence of the processes of IE formation and recombination on the ring result in the realization of a stationary excited state of the condensate, which is not spatially uniform. The indirect excitons are treated as genuine Bose particles. In addition, we suppose that the radial profile of the condensate density is governed by the processes which have no direct relation to the ring fragmentation. This allows us to consider a quasi one-dimensional problem.

2. Polar angle dependence of the condensate density. The Hamiltonian for the exciton system with the source reads

$$
\begin{aligned}
& \hat{H}=\hat{H}_{J}+\int d \vec{r} \hat{\Psi}^{+}(\vec{r}, t) \frac{\hat{p}^{2}}{2 m} \hat{\Psi}(\vec{r}, t)+ \\
& \frac{1}{2} \int d \vec{r} d \vec{r}^{\prime} \hat{\Psi}^{+}(\vec{r}, t) \hat{\Psi}^{+}\left(\vec{r}^{\prime}, t\right) U\left(\vec{r}-\vec{r}^{\prime}\right) \hat{\Psi}\left(\vec{r}^{\prime}, t\right) \hat{\Psi}(\vec{r}, t) .
\end{aligned}
$$

Here $\hat{\Psi}(\vec{r}, t)$ is a Bose field operator obeying the commutation relations $\left[\hat{\Psi}\left(\vec{r}_{1}, t\right), \hat{\Psi}^{+}\left(\vec{r}_{2}, t\right)\right]=\delta\left(\vec{r}_{1}-\vec{r}_{2}\right)$, $[\hat{\Psi}, \hat{\Psi}]=\left[\hat{\Psi}^{+}, \hat{\Psi}^{+}\right]=0$. For simplicity, we suggest that the repulsive pair interaction is local, i.e., $U(\vec{r})=\lambda \delta(\vec{r})$ and $\lambda>0$. In fact, this means either the Born approximation for the potential $U(r)$ with $\lambda=\int U(r) d \vec{r}$, or the gas approximation $n a_{2 D}^{2} \ll 1$, where $n$ is the twodimensional IE density and $a_{2 D}=m \lambda /\left(4 \pi \hbar^{2}\right)$ is an exact $s$-scattering length [11]. The contribution of the source is given by

$$
\hat{H}_{J}=\int d \vec{r}\left(J(t) \hat{\Psi}^{+}(\vec{r}, t)+J^{*}(t) \hat{\Psi}(\vec{r}, t)\right),
$$

where $J(t)=|J| e^{i \alpha(t)}$. It is natural to assume that the time dependence of the source is the same as for the wave function of the ground condensate state in the lack of the source, $\alpha(t)=-\mu t$. We suggest that the quantities $|J|$ and $\mu$ are time-independent.

Let us consider the equation of motion for $\hat{\Psi}$-operator, $i \hbar \partial \hat{\Psi}(\vec{r}, t) / \partial t=[\hat{\Psi}(\vec{r}, t), \hat{H}]$, assuming zero temperature of the system. Here, the most fraction of excitons is in the Bose-condensate state and thus we neglect noncondensate particles. Then $\hat{\Psi}$-operator becomes the $c$-number and obeys the equation

$$
i \hbar \dot{\Psi}=-\frac{\hbar^{2}}{2 m} \nabla^{2} \Psi+\lambda|\Psi|^{2} \Psi+J,
$$

which for $J=0$ turns into the Gross-Pitaevskii equation. Supposing that the radial profile of the condensate density is unvaried, we consider a quasi $1 \mathrm{D}$ problem. Then, the genuine density "in amplitude" refers to the result obtained as $n_{\text {true }} / n \sim \bar{a}_{\text {ex-ex }} / \Delta R \sim 10^{-2}$. For the polar frame with the origin at the ring center $\nabla^{2} \approx R^{-2} \partial^{2} / \partial \phi^{2}, d \vec{r} \approx 2 \pi R \Delta R d \phi=S d \phi$, where $\phi$ is the polar angle, $R$ is the ring radius, and $R \neq R(n)$. The aim of the work is to find a qualitative behavior $n(\phi)$ at various magnitudes of the parameters.

If one measures energy in units $E_{R}=\hbar^{2} /\left(2 m R^{2}\right)$, time in $\tau_{R}=\hbar / E_{R}$, and density in $S^{-1}$, the constants in Eq.(3) are dimensionless

$$
i \dot{\Psi}=-\Psi_{\phi \phi}^{\prime \prime}+\lambda|\Psi|^{2} \Psi+J .
$$

Let us seek for the solution (44) as $\Psi=\rho e^{i f}$, where $\rho^{2} \approx$ $n$ and $f$ are the density and phase of the condensate, respectively. Then, for the real and imaginary parts of $\Psi$ the following equations are valid

$$
\begin{array}{r}
\rho \dot{f}+\rho\left(f_{\phi}^{\prime}\right)^{2}-\rho_{\phi \phi}^{\prime \prime}+\lambda \rho^{3}+|J| \cos (\alpha-f)=0, \\
\dot{\rho}+2 \rho_{\phi}^{\prime} f_{\phi}^{\prime}+\rho f_{\phi \phi}^{\prime \prime}-|J| \sin (\alpha-f)=0 .
\end{array}
$$

Multiplying both sides of Eq.(6) by $2 \rho$ and integrating over $\phi$, we obtain that the rate of variation of the particle number $N$ equals

$$
\dot{N}=\int d \vec{r} 2 \rho \dot{\rho}=\int d \vec{r} 2 \rho|J| \sin (\alpha-f) .
$$

In what follows, we are interested in the stationary currentless states alone: $\dot{N}=0, f_{\phi}^{\prime}=0$. Then $f=\alpha+\pi l, l$ is an integer. So,

$$
\rho_{\phi \phi}^{\prime \prime}+\mu \rho-\lambda \rho^{3}-|J|(-1)^{l}=0,
$$

in which $\alpha(t)=-\mu t$ is taken into account. In the case of the homogeneous density $\left(\rho_{\phi}^{\prime} \equiv 0, \rho=\rho_{0}\right)$ and $|J|=0$, $\mu$ has a meaning of the chemical potential of the system:

$$
\mu=\lambda \rho_{0}^{2} .
$$

Note that for $|J|=0$ even in the unhomogeneous case the quantity $\mu$ can literally be treated as a chemical potential for the ground state of the system. In this case it can be introduced via $\Psi(t) \sim \exp (-i \mu t)$.

For $J \neq 0$ the uniform density is determined from the equation

$$
\mu \rho_{0}-\lambda \rho_{0}^{3}-|J|(-1)^{l}=0 .
$$

Employing the Cardano formulae, one can find from the analysis that there exist three regions in which: (i) homogeneous solution is absent (even $l, \mu<\mu_{*} \equiv$ $\left.3 \lambda(|J| /(2 \lambda))^{2 / 3}\right)$, (ii) homegeneous solution is unique (even $l, \mu=\mu_{*}$, and odd $l$ at $\mu \leqslant \mu_{*}$ ), and (iii) there exist 1 or 2 various homogeneous solutions (arbitrary $l$, $\left.\mu>\mu_{*}\right)$.

For the unhomogeneous case $\rho_{\phi}^{\prime} \neq 0$, the first integral of Eq.(8) is given by

$$
\left(\rho_{\phi}^{\prime}\right)^{2}=\frac{1}{2} \lambda \rho^{4}-\mu \rho^{2}+2|J|(-1)^{l} \rho+C .
$$

Here $C$ is a constant independent of $\phi$. Substituting $\rho=$ $\rho_{0} \cdot x$ and integrating, we have

$$
\int \frac{d x}{\sqrt{x^{4}-2 \mu_{\lambda} x^{2}+2 J_{\lambda} x+C_{\lambda}}}= \pm \sqrt{\frac{1}{2} \lambda \rho_{0}^{2}}\left(\phi-\phi_{0}\right) \equiv \tilde{\phi},
$$


where $\mu_{\lambda}=\frac{\mu}{\lambda \rho_{0}^{2}}, \quad J_{\lambda}=\frac{|J|(-1)^{l}}{\frac{1}{2} \lambda \rho_{0}^{3}}, \quad C_{\lambda}=\frac{C}{\frac{1}{2} \lambda \rho_{0}^{4}}$. Let $x_{1}, x_{2}, x_{3}$, and $x_{4}$ be the roots of equation $x^{4}-2 \mu_{\lambda} x^{2}+$ $2 J_{\lambda} x+C_{\lambda}=0$. From (12) it follows

$$
F\left(\frac{\sqrt{x_{1}-x_{3}} \sqrt{x_{2}-x}}{\sqrt{x_{1}-x_{2}} \sqrt{x_{3}-x}}, k\right)=-a \tilde{\phi},
$$

where $a=\frac{1}{2} \sqrt{x_{4}-x_{2}} \sqrt{x_{1}-x_{3}}, \quad k=\frac{\sqrt{x_{1}-x_{2}} \sqrt{x_{4}-x_{3}}}{\sqrt{x_{4}-x_{2}} \sqrt{x_{1}-x_{3}}}$. Here $F(x, k)$ is the Jacobi elliptic integral of the first kind with modulus $k$. Solving the latter equation in $x$, we arrive at

$$
x=\frac{x_{2}\left(x_{3}-x_{1}\right)+x_{3}\left(x_{1}-x_{2}\right) s n^{2}(a \tilde{\phi}, k)}{x_{3}-x_{1}+\left(x_{1}-x_{2}\right) s n^{2}(a \tilde{\phi}, k)},
$$

where $\operatorname{sn}(x, k)$ is the Jacobi elliptic sine with modulus $k$. (Note that the quantities $x_{1}, x_{2}, x_{3}, x_{4}$ should be chosen so that $x \geqslant 0, a^{2}>0$ and $0 \leqslant k^{2} \neq 1$. Otherwise, this unhomogeneous solution does not exist.) For $k \longrightarrow 1$, the period of $s n^{2}(x, k)$ tends to infinity and the density is independent of the polar angle. For $k \longrightarrow 0, s n^{2}(x, k) \longrightarrow \sin ^{2}(x)$. The position of the extrema of function $x(\phi)$ is determined by the quantity $\xi=\left(x_{1}-x_{2}\right)\left(x_{2}-x_{3}\right)\left(x_{1}-x_{3}\right)$. If $\xi=0, x_{\phi}^{\prime} \equiv 0$. For $\xi>0$, the points of minima $\tilde{\phi}_{l}^{\text {min }}=\frac{K(k)}{a} 2 l, x\left(\tilde{\phi}_{l}^{\text {min }}\right)=$ $x_{2}$ and maxima $\tilde{\phi}_{l}^{\max }=\frac{K(k)}{a}(2 l+1), x\left(\tilde{\phi}_{l}^{\max }\right)=x_{1}, l$ being an integer. For $\xi<0$, in these formulae one should replace $\tilde{\phi}_{l}^{\min } \rightleftarrows \tilde{\phi}_{l}^{\max }$.

It is seen that, in the general case, the condensate density $n(\phi)=\rho_{0}^{2} x^{2}(\phi)$ is a periodic function (see Fig. 1). The solution obtained allows us to find the period of density oscillations or, this is the same, the angular size of a fragment

$$
\Delta \phi_{f r}=\frac{2 K(k)}{a \sqrt{\frac{1}{2} \lambda \rho_{0}^{2}}},
$$

where $K(k)$ is the complete elliptic integral. The number of fragments at the circumference of the ring $N_{f r}=\frac{2 \pi}{\Delta \phi_{f r}}$ should be a positive integer. One can, in principle, find the constant $C=C(R)$ from this condition.

Note that the spatially unhomogeneous solutions are also possible in the lack of the source $(|J|=0)$. Then

$$
n(\phi)=\left(\rho_{0} x_{1}\right)^{2} s n^{2}\left(\sqrt{\frac{1}{2} \lambda \rho_{0}^{2}}\left(\phi-\phi_{0}\right) x_{2}, k_{0}\right),
$$

where $k_{0}=\frac{x_{1}}{x_{2}}, x_{1,2}^{2}=\mu_{\lambda} \pm \sqrt{\mu_{\lambda}^{2}-C_{\lambda}}$.

3. Conclusions and discussion. To conclude, we have obtained a set of solutions. All of them, both homogeneous and unhomogeneous, correspond to the minimum of the energy's functional of the system, i.e., to the stationary solutions (see Appendix). This simple theory

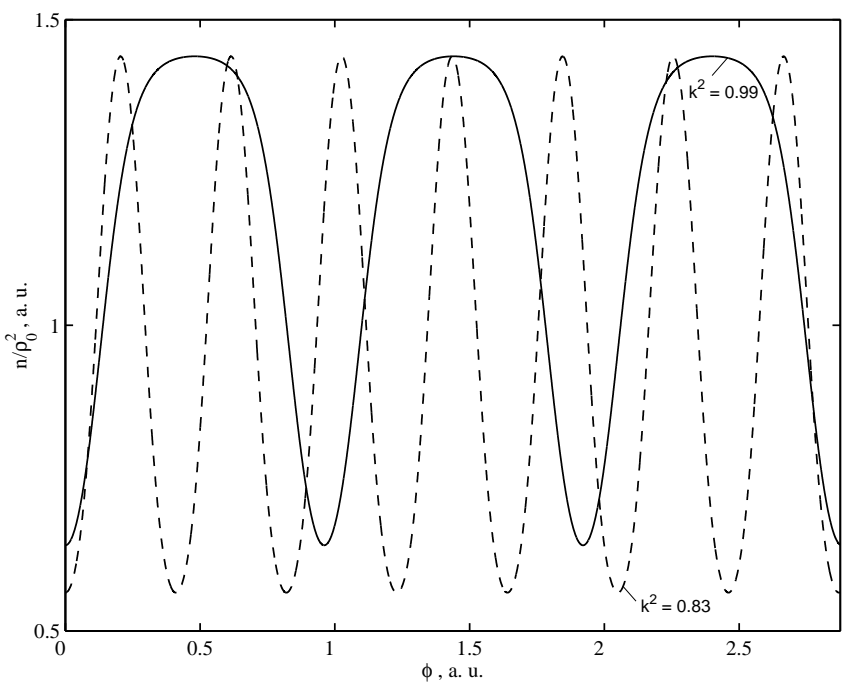

Figure 1: The examples of the behavior of the condensate density $n$ versus polar angle $\phi$ for $x_{1}=1.2, x_{2}=0.8, x_{3}=0.4$, $x_{4}=1.21$ (solid line) and for $x_{1}=1.2, x_{2}=0.75, x_{3}=0.6$, $x_{4}=2.1$ (dashed line).

cannot uniquely predict what a solution from the set will be realized in fact. However, it is clear that the spatially homogeneous solution (if it exists at $J \neq 0$ ) refers to the absolute minimum of energy and unhomogeneous ones correspond to the steady excited states of the condensate.

In experiments [1, 2] the IE formation-recombination processes lead to the condensate density fluctuations in the radial direction. As is shown in Ref. [12], at zero temperature in the cigar-shaped condensate due to the parametric resonance there takes place one-way transfer of energy from the transverse (radial) modes to the longitudinal ones. Closing this "cigar" in a ring, we come to the point that the spatially non-uniform distribution of the condensate density is nothing else than a standing wave corresponding to the macroscopically filling of the longitudinal modes. The consideration of the standing wave as a steady excited state of the condensate agrees with the experimental fact that the existing fragmentation starts to vanish with decreasing the pumping power but for all that the ring radius would vary negligibly.

Note that the fragmentation of the exciton ring can be explained in another way, assuming that the IEs are in the condensate state. Since the "ground" state of the exciton system is degenerate over the IE spin configurations, it represents a mixture of the condensates with different coupling constants $\lambda_{i j}>0$ for the mixture components (here $i, j$ are the indexes of the mixture components). As is known [13, 14], if for $i \neq j$ the condition $\lambda_{i j}^{2}>\lambda_{i i} \lambda_{j j}$ is fulfilled, the mixture components separate from each other in space. (On the observation of this effect in atomic condensates see, e.g., Ref. [15].) It is possible that such a separation for the ring geometry is observed in the experiments. 
Recently, the observations similar to MOCS ones have been reported in Ref. [16]. The microscopic derivation (starting with the electron-hole Hamiltonian) of Eq. (3) with $J=0$ is the same as in Ref. [17].

\section{Acknowledgments}

The authors are thankful to V.S. Babichenko for the suggestion of the form of the "source-drain" Hamiltonian and helpful comments in the early stage of the work. We also thank L.A. Maksimov for valuable discussions. The work is supported by the Russian Foundation for Basic Research.

Appendix. The total average energy of the system has form

$$
E[\rho]=\frac{1}{2 \pi} \int_{0}^{2 \pi} d \phi\left(\left(\rho_{\phi}^{\prime}\right)^{2}-\mu \rho^{2}+\frac{1}{2} \lambda \rho^{4}+2|J|(-1)^{l} \rho\right) .
$$

The minimum of the functional $E[\rho]$ is determined by Eq.(8) and equals

$$
E_{\min }[\rho]=\frac{1}{2 \pi} \int_{0}^{2 \pi} d \phi\left(2\left(\rho_{\phi}^{\prime}\right)^{2}-C\right)
$$

where $C$ is the constant from Eq.(11). In the case of the homogeneous density, involving Eq.(10), we arrive at $E_{\min }\left[\rho_{0}\right]=\mu \rho_{0}^{2}-\frac{3}{2} \lambda \rho_{0}^{4} \equiv-C_{0}$. This corresponds to the absolute energy minimum provided that

$$
\frac{1}{2 \pi} \int_{0}^{2 \pi} d \phi\left(\rho_{\phi}^{\prime}\right)^{2}>\frac{1}{2}\left(C-C_{0}\right)
$$

\section{References}

[1] L.V. Butov, A.C. Gossard and D.S. Chemla, Nature 418 (2002) 751.

[2] L.V. Butov, J. Phys.: Condens. Matter 16 (2004) R1577R1613.

[3] L.V. Butov, L.S. Levitov, B.D. Simons et al., Phys. Rev. Lett. 92 (2004) 117404.

[4] R. Rapaport, G. Chen, D. Snoke et al., Phys. Rev. Lett. 92 (2004) 117405.

[5] S. Denev, S.H. Simon, D.W. Snoke, Solid State Commun. 134 (2005) 59.

[6] Sen Yang, A.T. Hammack, M.M. Fogler et al., cond-mat/0606683

[7] L.S. Levitov, B.D. Simons and L.V. Butov, Phys. Rev. Lett. 94 (2005) 176404.
[8] V.I. Sugakov, Solid State Commun. 134 (2005) 63.

[9] C.S. Liu, H.G. Luo, W.C. Wu, cond-mat/0604564.

[10] Sen Yang, A.V. Mintsev, A.T. Hammack et al., cond-mat/0608342.

[11] L.P. Pitaevskii, Physics-Uspekhi 41 (1998) 641.

[12] Yu. Kagan, L.A. Maksimov, JETP Lett. 78 (2003) 734.

[13] E. Timmermans, Phys. Rev. Lett. 81 (1998) 5718.

[14] P. Ao and S.T. Chui, Phys. Rev. A 58 (1998) 4836.

[15] J. Stenger, S. Inouye, D.M. Stamper-Kurn et al., Nature 396 (1998) 345.

[16] A.V. Gorbunov, V.B. Timofeev, JETP Lett. 83 (2006) 146.

[17] A.I. Bezuglyj, S.I. Shevchenko, cond-mat/0612321. 